\title{
Functionalized Graphene as Cryo-EM Supporting Film
}

Jungjae Park ${ }^{1}$, Hyeongseop Jeong ${ }^{2}$, Euijun Choi $^{2}$, Jaekyung Hyun ${ }^{2,3}$ and Jong Min Yuk ${ }^{1}$

1. Department of Materials Science and Engineering, Korea Advanced Institute of Science and Technology, Daejeon, Republic of Korea

2. Electron Microscopy Research Center, Korea Basic Science Institute, Chungcheongbuk-do, Republic of Korea

3. Department of Bio-analytical Science, Korea University of Science and Technology, Daejeon, Republic of Korea

Recent developments in the electron microscopy equipment, including aberration correctors and direct electron detectors, and image processing algorithms pivoted dramatic advancement of cryo-electron microscopy (cryo-EM) single particle analysis. However, cryo-EM still suffers from electron beaminduced movement of specimen and sometimes lack of diverse particle orientation that is critical for 3D reconstruction. To resolve these issues, researches have attempted to apply additional supporting film to the holey carbon films during cryo-EM specimen preparation [2-4]. Recently, graphene oxide film is commonly used as supporting film, but graphene oxide is electrical insulator, too thick when multiple layers are formed, and deposition on the grid is usually inconsistent. So, in terms of supporting film, the graphene is most desirable material because graphene not only has high electron-transparency, minimal inelastic cross-section, high electrical/thermal conductivity, and mechanical strength but also layer number and coverage can be easily controlled [5]. However, graphene is not widely applied to biological electron microscopy since the nonpolar and hydrophobic properties prevent reliable protein absorption. In this work, we have used UV/Ozone and hydrogen plasma treatment to make oxidized graphene or hydrogenated graphene, respectively. We confirm that both treatments enhance the hydrophilicity and protein absorption on the graphene film.

We synthesis the monolayer graphene to minimize the background noise by using chemical vapor deposition process, followed by direct transfer of the graphene to the gold holey-carbon Quantifoil TEM grid. We firstly apply UV/Ozone treatment to form the epoxide group on the graphene surface and consequently graphene become negatively charged at the terminal. Second, we apply the low energy hydrogen plasma treatment to form hydrogen bond on the graphene surface and hence graphene become positively charged at the terminal. Figures 1(a) and (b) show the contact angle between graphene film and water droplet. The graphene film becomes hydrophilic when graphene is oxidized or hydrogenated. Electron micrographs in Figure 1(c) show that the graphene is intact after the treatments, indicating that the treatments are nondestructive to the graphene. We tested these functionalized graphene films on cryo-EM experiment using $20 \mathrm{~S}$ proteasome. Cryo-electron micrographs in Figure 2(a) show 20S proteasome particles preserved in empty hole and the particles adsorbed onto untreated, oxidized, and hydrogenated graphene films. The oxidized or hydrogenated graphene film improve both the protein adsorption by $2 \sim 3$ times and orientation distribution, as shown in Figure 2(a).

In summary, we functionalized the graphene with UV/Ozone or the hydrogen plasma treatment and applied this functionalized graphene to cryo-EM. The use of graphene film may facilitate 3D reconstruction by reducing the beam induced motion and improving distribution of particle orientations. Our results suggest that the functionalized graphene film is a promising alternative to previous cryo-EM sample preparation methods [6]. 
References:

[1] Sjors HW Scheres, J. Struct. Biol. 177 (2012), p. 630.

[2] R. S. Pantelic et al, Nano Lett. 11 (2011), p. 4319.

[3] R. S. Pantelic et al, J. Struct. Biol. 170 (2010), p. 152.

[4] R. S. Pantelic et al, J. Struct. Biol. 174 (2011), p. 234.

[5] Christopher J Russo and Lori A Passmore, Nat. Met. 11 (2014), p. 649.

[6] The authors acknowledge funding from Samsung Research Funding Center of Samsung Electronics under Project Number SRFC-IT1701-00.

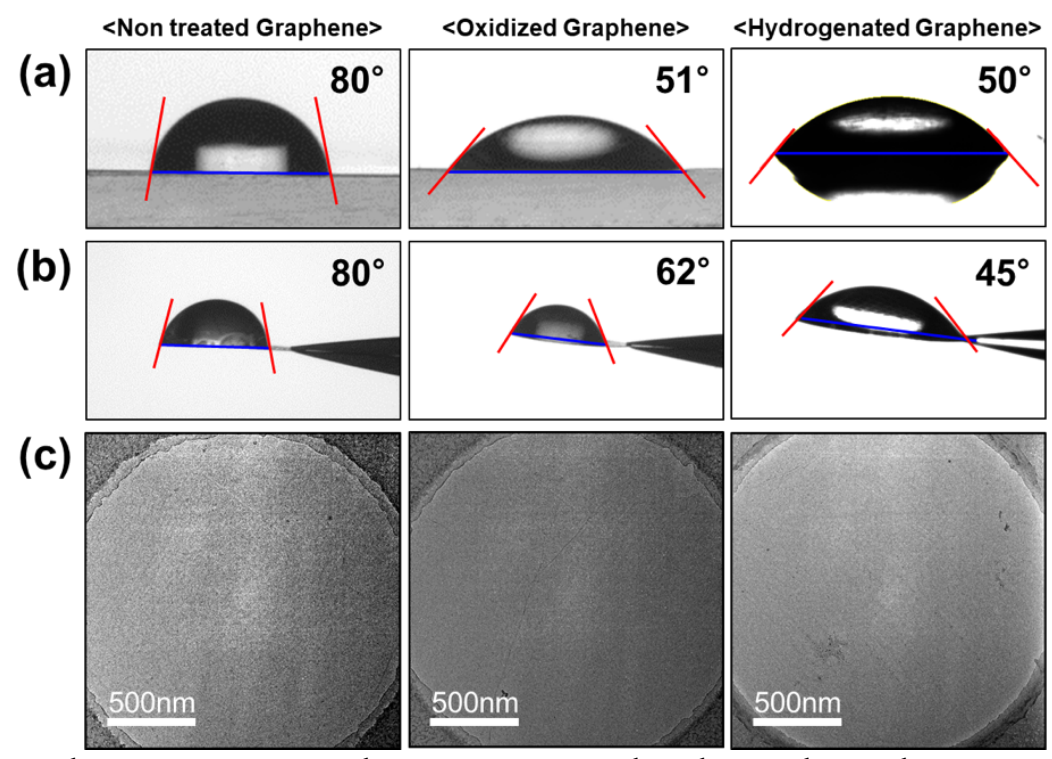

Figure 1. (a) Contact angle measurements between water droplet and graphene on $\mathrm{Cu}$ foil. (b) Contact angle measurements between water droplet and graphene on TEM grid. (c) Electron micrographs of graphene film deposited on the holey-carbon TEM grids.
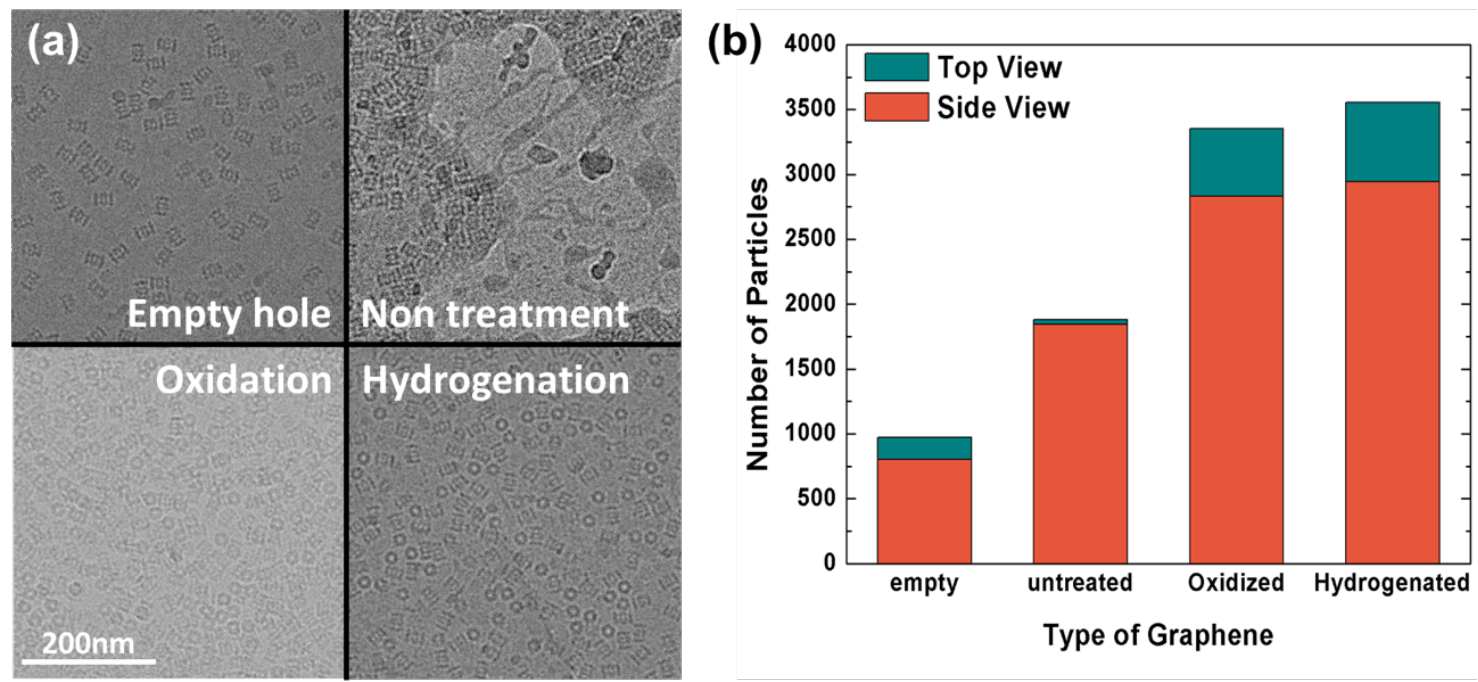

Figure 2. (a) Cryo-electron micrographs of $20 \mathrm{~S}$ proteasome trapped in empty hole and the particles adsorbed onto nontreated, oxidized, and hydrogenated graphene, respectively. (b) A graph showing the distribution of particle orientations. 